\title{
Experimental Study on a Hybrid Control Method using Neural Networks with Particle Swarm Optimization
}

\author{
Shenglin $\mathrm{Mu}^{\mathrm{a},{ }^{,} \text {, Kanya Tanaka }}{ }^{\mathrm{b}}$, Shota Nakashima ${ }^{\mathrm{b}}$, Shingo Aramaki ${ }^{\mathrm{b}}$, \\ Hiromasa Tomimoto $^{\mathrm{b}}$, Hiroshi Shibata ${ }^{\mathrm{a}}$, Hiroshi Ohwada ${ }^{\mathrm{a}}$, Shinichi Imai ${ }^{\mathrm{a}}$ \\ ${ }^{a}$ National Institution of Technology, Hiroshima College, 4272-1, Higashino, \\ Osakikamijima-cho, Toyota-gun, Hiroshima, 725-0231, Japan \\ ${ }^{\mathrm{b}}$ Graduate School of Science and Engineering, Yamaguchi University, \\ 2-16-1, Tokiwadai, Ube, 755-8611, Japan
}

*Corresponding Author: mshenglin@ @iroshima-cmt.ac.jp

\begin{abstract}
Artificial intelligence (AI) is widely applied in industrial products and innovations. In control engineering, the application of AI opened a novel area of intelligent control. Since then the famous algorithms of AI, such as Neural Network (NN) and Genetic Algorithm (GA) have been being researched, developed and applied for various control applications. Alone with optimization algorithms introduced in intelligent control, more and more hybrid methods have been developed. In this research, we introduce a hybrid intelligent method, based on NN combined with Particle Swarm Optimization (PSO), to the auto-tuning of variable gain PID control in real-time environment. The proposed method is with simple structure and capable to improve the control performance of traditional PID control. In this paper, the control performance and effectiveness of the proposed method was studied according to experimental results on the servo system of ultrasonic motor.
\end{abstract}

Keywords: intelligent control, neural network, particle swarm optimization, ultrasonic motor, positioning control.

\section{Introduction}

Alone with the development of Artificial Intelligence (AI), advanced intelligent algorithms are expected to play important roles in various kinds of innovations and industrial products. Decades before, the applications of AI in control engineering brought us a novel area of intelligent control. There have been being many famous intelligent algorithms, such as Neural Networks (NN), Genetic Algorithms (GA) and Fuzzy and so on, introduced to control methods with a great perspective. They have been being researched, developed and improved, giving important progress to control technology. Based on intelligent method of NN, in this paper, we make further study on an intelligent control method combined with Particle Swarm Optimization (PSO) for variable gain PID control.

In our proposed method, $\mathrm{NN}$ is introduced as intelligent tuning unit for PID control. Comparing with GA and Fuzzy, NN performs quick and effective in control applications. The technology using NN has been introduced as control method in electronic products around us. However, in conventional NN with back propagation (BP) method for weight updating, there is an estimation of Jacobian which cannot be estimated based on specific applications, but decided according to experience. For avoiding the estimation problem, PSO is implemented in the proposed method for constructing a hybrid intelligent PID control method. PSO is an optimization method originated from the model of swarm of animals, fishes and insects. Since the model of PSO is simple and the estimation in it is easy, the algorithm is developed quite fast in recent years. In the proposed hybrid method, PSO is introduced for the weight updating in NN. The updating procedure requires only error signal of the system, without any modeling information of the system. The mechanism of the hybrid method is simple and easy to use.

To confirm the effectiveness of the proposed method, some basic work have been done in previous research. In 
this paper, we will report some experimental results for understanding the method better. On a servo system of Ultrasonic Motor (USM) with characteristic changes and non-linearity in its running, the control performance of the proposed method was evaluated according to experimental results. The paper is organized as follows. The PID control for USM is introduced in Sect. 2. In Sect. 3, the proposed intelligent method of hybrid NN with PSO is introduced. Section 4 studies the experiments and results. Conclusions are given in Sect. 5 .

\section{PID Control for Ultrasonic Motor}

\subsection{Ultrasonic motor}

As a plant in the study, a Travelling Wave Ultrasonic motor (TWUSM) was introduced in the research. Different from traditional electromagnetic motors, USM is driven by friction force. The driving theory of USM is shown in Fig. 1.

The concept of friction driven motor was proposed quite early. However, the motor was not realized in the early age since it was proposed. It was commercialized in 1980 's by Mr. Sashida ${ }^{(1)}$. USM was found with very outstanding features since it was commercialized. Firstly, USM offers higher torque comparing with electromagnetic motor in the same size. Secondly, USM can give high torque in low speed range without any gear, and in no power condition, can give high retention torque. Thirdly, since USM is driven by friction generated from ultrasonic vibration, it runs very quiet offering high accuracy. Meanwhile, there is an important feature that owing to the special friction driven principle comparing with other electromagnetic motors, USM generates no electromagnetic noise and has no interferences to other electronic devices. Therefore, USM is capable to be applied in watches,

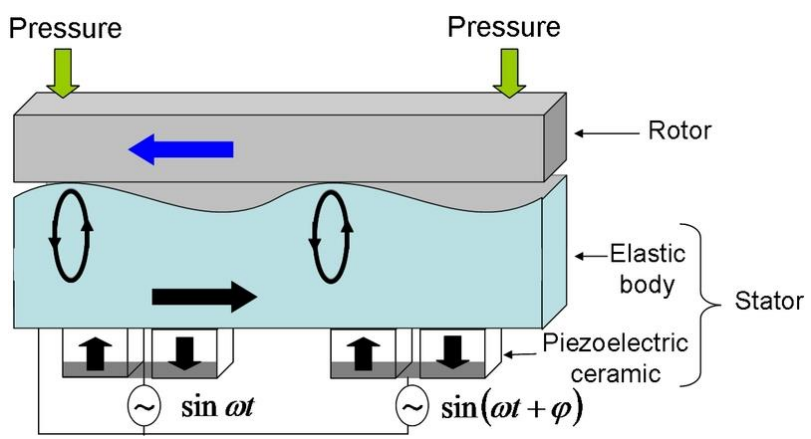

Fig. 1. Driving theory of USM. autofocus cameras, cell phones, and so on. USM is still expected to be employed in more and more applications. Especially, owing to its Electromagnetic Compatibility (EMC), it is expected to be introduced more in medical and welfare fields ${ }^{(2,3)}$.

However, there are still many difficulties in the applications of USM. Firstly, the life duration of USM is not long owing to abrasion caused by the friction driving. Secondly, special driving system which is more complex than that of traditional electromagnetic motors has to be applied. For control of USM, the characteristics of USM will change according to condition changes of temperature, humidity and load and so on. Meanwhile, since USM is driven by friction force, there is non-linearity in the control of it. There are no exact control models of USM.

\subsection{PID control for ultrasonic motor}

The basic scheme of PID control for USM can be illustrated as Fig. 2 shows. In the scheme, $r(k), u(k)$, and $y(k)$ are the object input, the input and the output, respectively. $G_{P I D}\left(z^{-1}\right)$ is the PID controller. The input $u(k)$ is synthesized as

$$
\begin{aligned}
& u(k)=u(k-1)+\left(K_{P}+K_{I}+K_{D}\right) e(k) \\
& -\left(K_{P}+2 K_{D}\right) e(k-1)+K_{D} e(k-2)
\end{aligned}
$$

where $K_{P}, K_{I}$ and $K_{D}$ are the gains of the PID controller. The error denoted by $e(k)$ expressed as

$$
e(k)=r(k)-y(k)
$$

The PID controller $G_{P I D}\left(z^{-1}\right)$ can be denoted as the following equation shows.

$$
G_{P I D}\left(z^{-1}\right)=\frac{K_{P}\left(1-z^{-1}\right)+K_{I}+K_{D}\left(1-z^{-1}\right)^{2}}{1-z^{-1}}
$$

In this research, the prosed intelligent method was proposed as Fig. 3 shows. The scheme is designed for tuning PID gains automatically to achieve good performance in USM control. In previous research, PID control which was proved to work well even without any

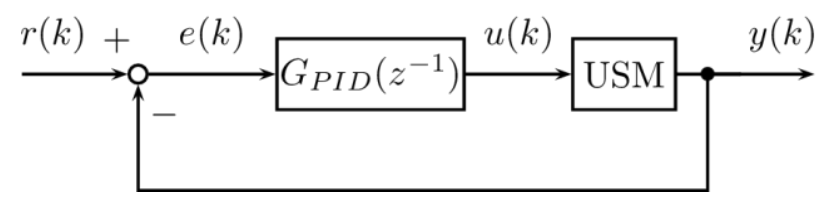

Fig. 2. Block diagram of PID control for USM. 
mathematic models employed.

\section{Intelligent PID control for ultrasonic motor}

In this paper, neural network has been introduced as the main intelligent unit based on PID control of ultrasonic motor. The proposed scheme is a fixed structure. The learning is designed to be implemented by PSO algorithm.

As introduced above, PSO is a global optimization algorithm discovered through the simulation of a simplified social model. Figure 4 shows a basic model of particle movement in PSO algorithm. $x_{i}(k)$ means the position of the $i_{\text {th }}$ particle in the $k$ step, $x_{i}(k-1)$ and $x_{i}(k+1)$ mean the position of the $(k-1)$ step and the $(k+1)$ step, respectively. gbest is the best position of the whole swarm, and pbest is the best position of the particle. $v_{i}(k)$, which expresses the position variance, is the velocity of the particle at the $k$ step. It is related to the best positions as the dash lines show in the figure. In NN learning, we define the value of weights as the position information contained by $N$ particles. The weights of NN are designed to be updated by an inertia weight approach (IWA) type PSO algorithm expressed in

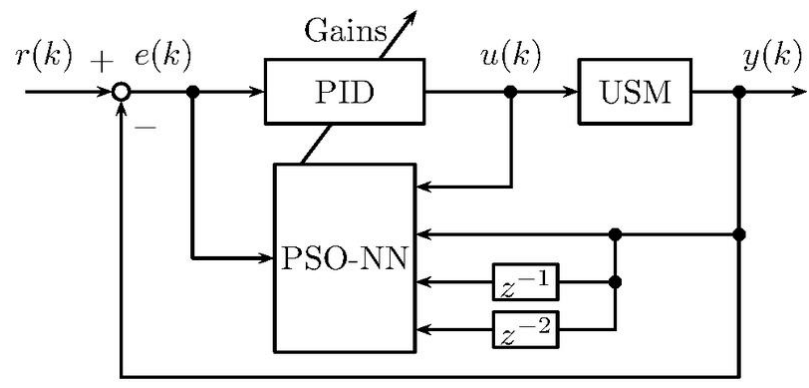

Fig. 3. Proposed scheme of PSO-NN method based on PID control for USM.

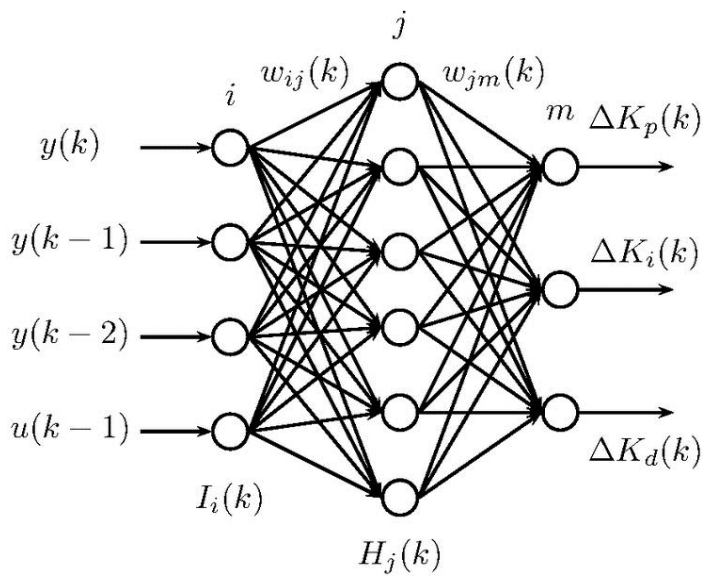

Fig. 4. Neuron structure in PSO-NN control unit.
Eq.(4) and Eq.(5).

$$
\begin{aligned}
& V_{n+1}=\tau \cdot V_{n}+c_{1} \cdot r_{1} \cdot\left(\text { pbest }_{n}-X_{n}\right) \\
& +c_{2} \cdot r_{2} \cdot\left(\text { gbest }_{n}-X_{n}\right) \\
& X_{n+1}=X_{n}+V_{n}
\end{aligned}
$$

In Eq.(4), $c_{1}$ and $c_{2}$ are two positive constants, $r_{1}$ and $r_{2}$ are two random numbers within [0,1]. In Eq.(5), the weight factor which balances the global and local search, is calculated as follows.

$$
\tau=\tau_{\max }-\frac{\tau_{\max }-\tau_{\min }}{\text { episode } \text { epx }_{\text {max }}} \cdot \text { ep }
$$

where episode means the number of current iteration, episode $_{\max }$ presents the max iteration number in the searching. $\tau_{\max }$ and $\tau_{\min }$ are the weight factor's maximum and minimum value, respectively ${ }^{(4-7)}$.

\section{Experiments and Study}

In this research the USM servo system with the USM, encoder, and the magnetic brake connected on a common axis was employed as shown in Fig. 5. The control signal will be input to the motor via the I/O board to the driving circuit. The fetched positioning signal from encoder will be recorded to the PC passing through I/O board.

In the experiments, a group of signal was employed set to run 10 cycles to evaluate the control performance on the servo system. Figure 6(a) shows the response of the fixed gain PID control method with the gains of $\mathrm{KP}=5.0, \mathrm{KI}=2.0$, $\mathrm{KD}=0.1$, in 3 cycles. It is clear that the output signal $y(t)$ follows the object input $r(t)$ in the runs. However, the error signal shown in Fig. 6(b) tells that there were some positioning error in the control process. Especially, the error were larger when the revolving speed varied quite fast. Figure 7(a) shows the response of the proposed PSO-NN type hybrid intelligent control method. There were no visible error in the response signal, the same as the response results

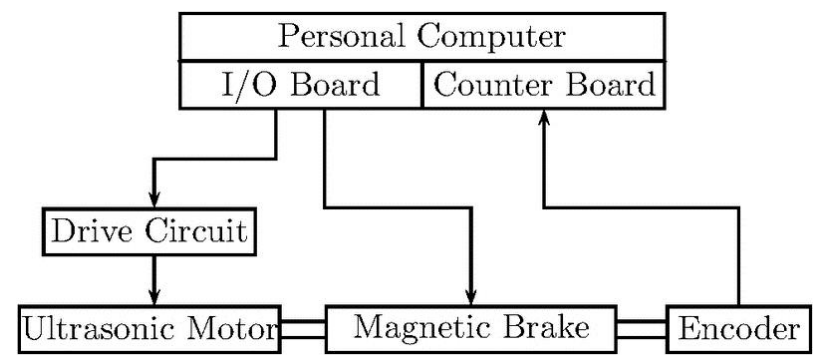

Fig. 5. Structure of USM servo system. 
in fixed gain PID control. However, comparing with the error signal shown in Fig. 6(b), the error signal of the

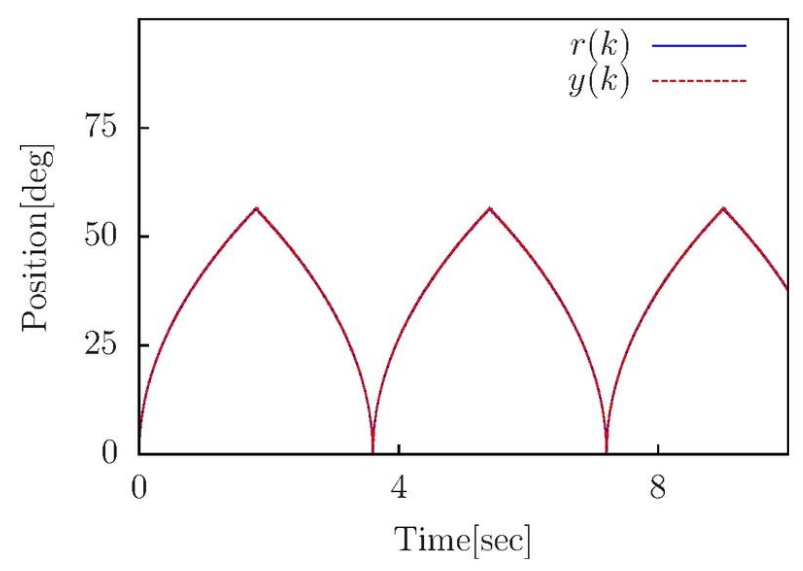

(a) Response of varied triangle signal input proposed method shown in Fig. 7(b) gave better positioning performance. Meanwhile, it cannot be ignored that the error

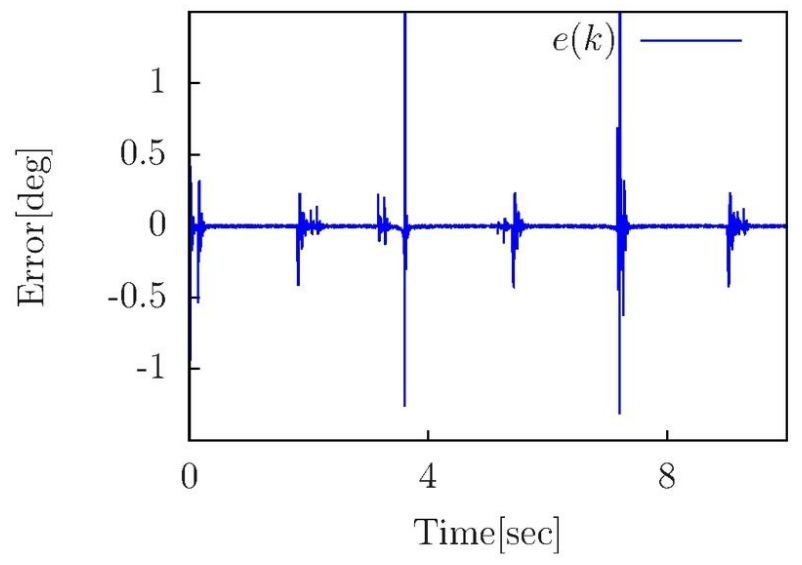

(b) Error signal of varied triangle signal response

Fig. 6. Experimental results of fixed gain type PID

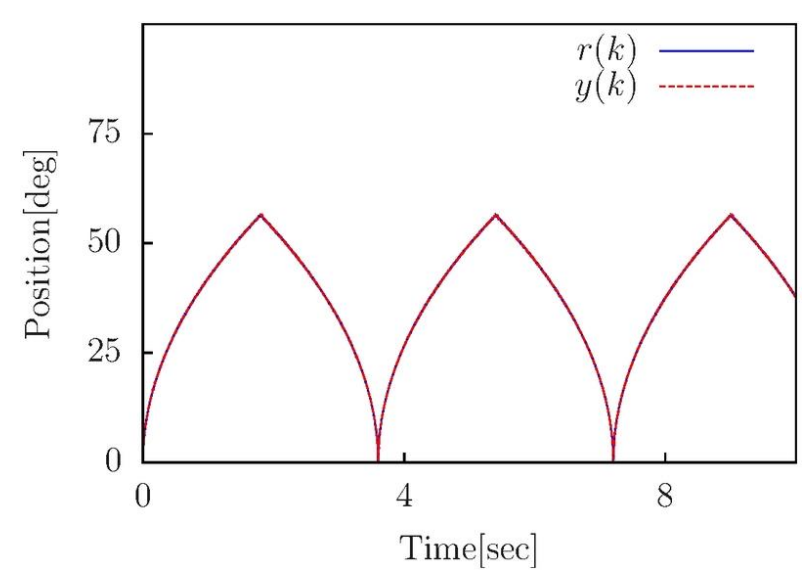

(a) Response of varied triangle signal input

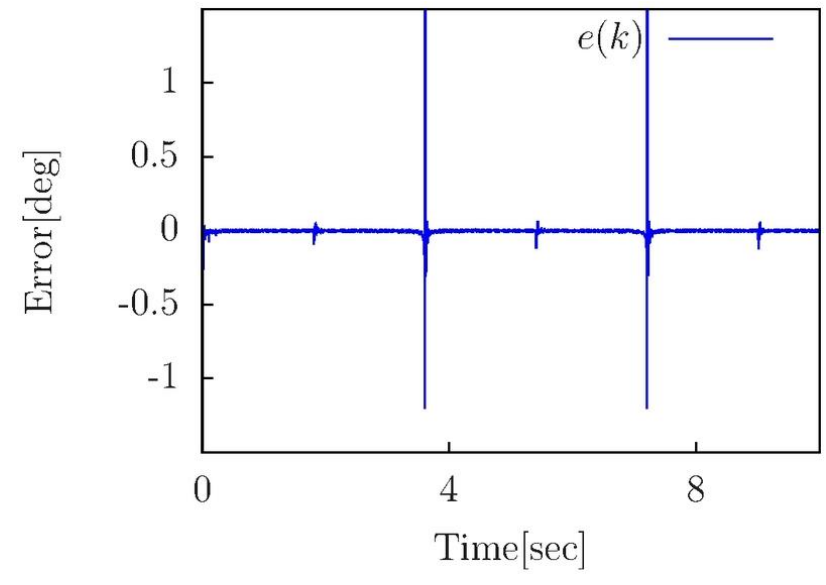

(b) Error signal of varied triangle signal response

Fig. 7. Experimental results of hybrid intelligent method using PSO-NN

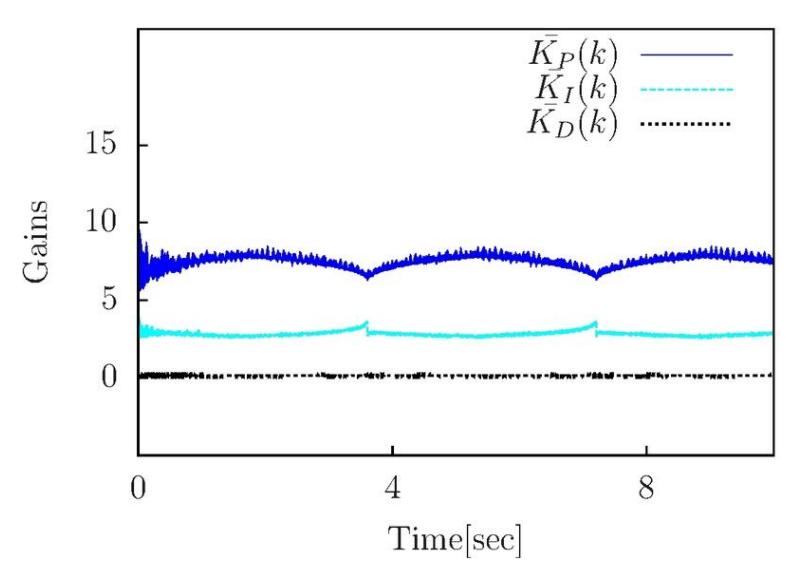

(a) Gain variation in the proposed method

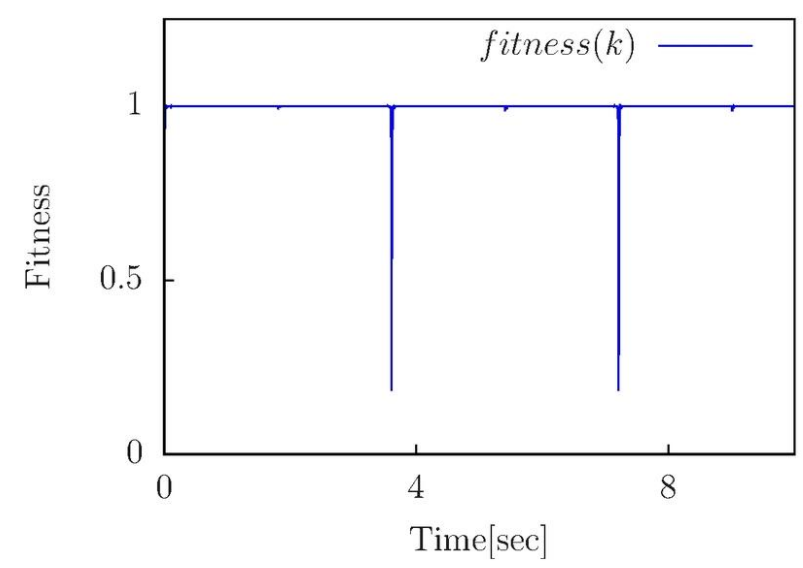

(b) Fitness variation in the propose method

Fig. 8. Parameter variation in the hybrid intelligent method using PSO-NN 
signal were still at the same level when the revolving speed varied with large acuteness. Therefore, the control performance is expected to be improved further in the following research. The parameter variation are shown in Fig. 8. Figure 8(a) shows the variation of PID gains in the real-time control. The continuous variation of gains offered better control performance than conventional fixed gain PID method. Meanwhile, the fitness in PSO algorithm, which is shown in Fig. 8(b), also leads to the same conclusion as the error signals showed above. The control performance is required to be improved in the range where the revolving speed varied suddenly.

\section{Conclusions}

According to the experimental results studied above, the effectiveness of the on the hybrid control method using PSO-NN was confirmed. The intelligent method is able to be applied in compensating characteristic changes USM in real-time control.

\section{References}

(1) T. Kenjo and T. Sashida: "An Introduction of Ultrasonic Motor" Oxford Science Publications, 1993.

(2) K. Adachi: "Actuator with friction drive: Ultrasonic motor", The Japan Society of Mechanical Engineers, Vol. 108: pp. 48-51, 2005

(3) C. Zhao: "Ultrasonic Motor - Technologies and Applications", Science Press Beijing and Springer-Verlag Berlin Heidelberg, Beijing, 2011

(4) J. Kennedy, R. Eberhart: "Swarm Intelligence", Morgan Kaufmann Publishers, 2001

(5) J. Kennedy and R. Eberhart: "Particle Swarm Optimization", Proc. IEEE Int. Conf. Neural Networks, Perth, Australia, pp. 1942-1948, 1995

(6) M. Clerc and J. Kennedy: "The Particle Swarm: Explosion, Stability, and Convergence in a Multi-Dimensional Complex Space", IEEE Trans. Evolutionary Computation, Vol. 6, No. 1, pp.58-73, 2002

(7) M. Ito and M. Tanaka: "A Study of Particle Swarm Optimization for Neural Network Training”, A publication of Electronics, Information and System Society, pp. 1087-1089, 2005 